\title{
The Edgeworth Conjecture with Small Coalitions and Approximate Equilibria in Large Economies
}

\author{
SIDDHARTH BARMAN, Indian Institute of Science, India \\ FEDERICO ECHENIQUE, California Institute of Technology, USA
}

\begin{abstract}
We revisit the connection between bargaining and equilibrium in exchange economies, and study its algorithmic implications. We consider bargaining outcomes to be allocations that cannot be blocked (i.e., profitably retraded) by coalitions of small size and show that these allocations must be approximate Walrasian equilibria. Our results imply that deciding whether an allocation is approximately Walrasian can be done in polynomial time, even in economies for which finding an equilibrium is known to be computationally hard.
\end{abstract}

CCS Concepts: • Theory of computation $\rightarrow$ Market equilibria.

Additional Key Words and Phrases: Walrasian Equilibrium, Core Convergence, Approximate Carathéodory Theorem, Piecewise-Linear Concave Utilities

\section{ACM Reference Format:}

Siddharth Barman and Federico Echenique. 2020. The Edgeworth Conjecture with Small Coalitions and Approximate Equilibria in Large Economies. In Proceedings of the 21st ACM Conference on Economics and Computation (EC '20), fuly 13-17, 2020, Virtual Event, Hungary. ACM, New York, NY, USA, 2 pages. https: //doi.org/10.1145/3391403.3399481

\section{EXTENDED ABSTRACT ${ }^{1}$}

We develop a quantitative core convergence theorem, with economic and algorithmic implications. Economists have, since Francis Edgeworth in 1881, been interested in the convergence of bargaining in large finite economies to market equilibrium. The celebrated Edgeworth conjecture states that bargaining indeterminacy and monopoly power disappear in large economies. Edgeworth focused on the contract curve, what we think of now as the core. Specifically the core of an economy is the set of allocations (redistribution of goods among agents) that no subset, or coalition, of agents can improve upon via re-trading among themselves. While the core requires agents to join coalitions of arbitrary size, in our quantitative core convergence theorem we show that coalitions of a fixed size suffice. The fixed size depends polynomially on the approximation error, the number of goods in the economy, and consumers' heterogeneity.

Edgeworth's conjecture was first formalized by Debreu and Scarf [3] as well as Aumann [1]. In our paper we shall follow Debreu and Scarf, ${ }^{2}$ who consider a large economy with limited heterogeneity. Their idea, which goes back to Edgeworth himself, is to postulate a finite number of agent types, say $h$, and then imagine a sequence of replica economies. The $n$-th replica of the economy has $n$ identical copies of each type of agent $t \in[h]$. Under standard assumptions, they show that, in the

\footnotetext{
${ }^{1}$ A full version of this paper is available at: https://arxiv.org/abs/1905.05165

${ }^{2}$ Aumann's model assumes a limit economy with a continuum of agents, hence there is no scope in his model to address the questions we are interested in.
}

Permission to make digital or hard copies of part or all of this work for personal or classroom use is granted without fee provided that copies are not made or distributed for profit or commercial advantage and that copies bear this notice and the full citation on the first page. Copyrights for third-party components of this work must be honored. For all other uses, contact the owner/author(s).

EC '20, July 13-17, 2020, Virtual Event, Hungary

(C) 2020 Copyright held by the owner/author(s).

ACM ISBN 978-1-4503-7975-5/20/07.

https://doi.org/10.1145/3391403.3399481 
limit as $n \rightarrow \infty$, the core of the $n$th replica economy approaches the set of Walrasian equilibrium allocations. Note that the core convergence theorem not only requires $n$ to be asymptotically large, but, to address the core, one has to account for coalitions that are arbitrarily large.

The economic contribution of our work in the form of a non-asymptotic core convergence theorem, for a relaxed notion of the core: one that only requires robustness to blocks by small coalitions. The algorithmic contribution is to the problem of testing whether an allocation is a (approximate) Walrasian equilibrium allocation.

The $\kappa$-core is the set of allocations that are not blocked by coalitions of size at most $\kappa$. We show that core convergence is obtained as long as $\kappa$ is polynomially large (in the approximation parameter and agent heterogeneity) and the size of the economy is at least $\kappa$. Thus, core convergence is obtained in finite economies. Specifically, we prove that in any replica economy with well-behaved utilities, allocations in the $\kappa$-core are, in fact, $\varepsilon$-Walrasian, as long as $\kappa$ is $O\left(\frac{h^{2} \ell}{\varepsilon^{2}}\right)$; here, $h$ denotes the heterogeneity of the economy (the number of different types of agents), $\ell$ denotes the number of goods, and $\varepsilon>0$ is the approximation parameter. Note that our result does not require the number of agents $n$, or the size of blocking coalitions, to be arbitrarily large.

Our work develops new techniques for addressing core convergence. Instead of relying on limiting, or measure-theoretic, arguments as in the classical literature, our proof builds upon geometric insights and dimension-free results. Specifically, we employ the approximate version of Carathéodory's theorem (see [2] and references therein).

Our key technical insight is provided through an efficiently implementable characterization of approximate Walrasian equilibrium allocations. Our characterization yields, not only the quantitative version of core convergence, but also an efficient algorithm that tests whether a given allocation can be supported as an approximate equilibrium. In particular, our algorithm takes as input an allocation, and determines whether there are prices that make the allocation into an approximate Walrasian equilibrium. This result is potentially useful to understand the policy objectives that can be obtained in a decentralized fashion, as a Walrasian equilibrium. It also contributes to the algorithmic literature on equilibrium computation, as it identifies a notable dichotomy between testing and computing Walrasian equilibria.

The above-mentioned results require the utilities in the economy to be strongly concave. Hence, as is, they do not address piecewise-linear concave (PLC) utilities, which, while concave, are not strongly concave. The PLC case is particularly important in light of its hardness results; see, e.g., [4]. However, we show that the developed ideas can be adapted to obtain an efficient testing algorithm even for PLC economies.

\section{ACKNOWLEDGMENTS}

Federico Echenique thanks the National Science Foundation for its support through grants SES1558757 and CNS-1518941. Siddharth Barman gratefully acknowledges the support of a Ramanujan Fellowship (SERB - SB/S2/RJN-128/2015) and a Pratiksha Trust Young Investigator Award.

\section{REFERENCES}

[1] Robert J Aumann. 1964. Markets with a continuum of traders. Econometrica (1964), 39-50.

[2] Siddharth Barman. 2015. Approximating nash equilibria and dense bipartite subgraphs via an approximate version of Caratheodory's theorem. In Proceedings of the forty-seventh annual ACM symposium on Theory of computing. ACM, 361-369.

[3] Gerard Debreu and Herbert Scarf. 1963. A limit theorem on the core of an economy. International Economic Review 4, 3 (1963), 235-246.

[4] Jugal Garg, Ruta Mehta, Vijay V Vazirani, and Sadra Yazdanbod. 2017. Settling the complexity of Leontief and PLC exchange markets under exact and approximate equilibria. In Proceedings of the 49th Annual ACM SIGACT Symposium on Theory of Computing. ACM, 890-901. 